\title{
Fuzzy Particle Swarm Optimization for Multilevel Thresholding in Image Segmentation and its Performance Evaluation with PSO
}

\author{
B.Sridevi ${ }^{1}$, N.Thambiraj ${ }^{2}$ and M.Chitra ${ }^{3}$ \\ \{ bsd.maths@psgtceh.ac.in ${ }^{1}$, kannanpsgtech12@gmail.com ${ }^{2}$, chitramarappan@gmail.com $\left.{ }^{3}\right\}$ \\ Associate Professor, Department of Mathematics, PSG College of Technology ${ }^{1}$, Assistant Professor, \\ Department of Mathematics, PSG Polytechnic College ${ }^{2}$, Student, MSc Applied Mathematics, PSG \\ College of Technology $y^{3}$
}

\begin{abstract}
In many applications of image processing, the gray levels of pixels belonging to an object are quite different from the gray levels of the pixels belonging to its background. The simplest property that pixels in a region can share is intensity. So, a natural way to segment such regions is through thresholding. This paper aims to determine the optimal threshold value using fuzzy systems in particle swarm optimization (PSO) algorithm. The proposed technique satisfies the objective function for Otsu method by maximizing between-class variance and also it overcomes the limitation of PSO. The efficiency and the stability of the algorithm is confirmed with few benchmark images.
\end{abstract}

Keywords: Fuzzy, PSO, Performance.

\section{Introduction}

A fundamental step in image processing is that images need to be segmented to identify their key components. The task of image segmentation is a first step in many computer vision methods that serves to simplify the problem by grouping the pixels in the image in logical ways. Thresholding is an important step in many computer vision tasks such as image segmentation. However, selection of the optimal threshold value often appears as a challenging problem to researchers.

Thresholding method is very commonly used to segment an image into two or more clusters $[1,2,3]$. Based on number of image segments thresholding techniques are either bilevel or multilevel [4]. Higher number of thresholds complicates the problem. So biologically inspired Particle Swarm Optimization (PSO) method have been used to solve this optimization problem [5, 6]. Many effective multi-level problem thresholding problem are proposed based on the issue of optimal thresholding [1, 9, 12, 18 19, 20, 21, 22]. For finding the optimal set of thresholds exhaustive search method based on the Otsu criterion [9] is proposed. Though the Otsu approach is simple it is computationally expensive. For $\mathrm{n}-1$ optimal thresholds the exhaustive search in [2] involves evaluations of fitness of $n(L-n+1)^{n-1}$ combinations of thresholds. So the method is not computationally effective.

Many biologically algorithms have been proposed in image segmentation [2 11, 12]. In situations where the function to be optimized is discontinuous, non-differentiable, or involves 
nonlinearly related parameters the conventional optimization techniques fails to find a satisfactory solution. In such cases bio-inspired algorithms gives a better optimal In [23, 24, 25, 36] In Particle Swarm optimization algorithm (PSO) [36] to increase the velocity of a particle a non-linear function is defined for modifying the weights. Though few bio-inspired methods sets the particles to converge to a local optimum and is also fast convergence but sometimes the fitness are unacceptable. To overcome the limitations of PSO adjustments to the parameters are made by incorporating fuzzy rules [26]. In Gaussian approach (GPSO) the inertia constant is no longer needed and the acceleration constants are replaced by random numbers with Gaussian distributions [27]. Latter fractional-order extension, fractional order Darwinian PSO (FODPSO) [ 2829 30, 31] was proposed. Improved PSO (IPSO) based multilevel thresholding algorithm is proposed to search the near optimal thresholds [34].

In this work to overcome the limitations of PSO algorithm, the parameters involved in the PSO algorithm are adjusted by framing appropriate fuzzy rules (FPSO) where the weights of social and cognitive influence are modified.

This paper is organized as follows: Section 2 discussses the basic concepts of thresholding in image segmentation. In Section 3 bio-inspired technique particle swarm optimization is presented.. In Section 4, the proposed fuzzy particle swarm optimization method is discussed and the experimental results are copared on three different standard images. The conclusion is presented in section 5 .

\section{Thresholding}

To cluster images a very commonly used technique in image segmentation is thresholding [2]. The fast and economical in computation of image segmentation process is histogram thresholding. Multilevel thresholding is highly effective to segment a gray level image into several distinct regions. This technique determines more than one threshold for the given image and segments the image into certain brightness regions, which correspond to one background and several objects.

In multi level thresholding the two-level thresholding is extended to generic n-level, $t_{c}^{j}$, $\mathrm{j}=1, \ldots, \mathrm{n}-1 \mathrm{n}-1$ threshold levels:

$$
F^{c}(x, y)=\left\{\begin{array}{cc}
0, & f^{c}(x, y) \leq t_{1}^{c} \\
\frac{1}{2}\left(t_{1}^{c}+t_{2}^{c}\right), & t_{1}^{c}<f^{c}(x, y) \leq t_{2}^{c} \\
\frac{1}{2}\left(t_{n-2}^{c}+t_{n-1}^{c}\right), & t_{n-2}^{c}<f^{c}(x, y) \leq t_{n-1}^{c} \\
L & f^{c}(x, y)>t_{n-1}^{c}
\end{array}\right.
$$

where $\mathrm{x}$ is the width (W) and $\mathrm{y}$ is height $(\mathrm{H})$ of the pixel of the image of size $\mathrm{H} \mathrm{x} \mathrm{W}$ denoted by $f^{c}(x, y)$ with intensity levels L in each RGB (red-green-blue) component in the range $0,1, \ldots, \mathrm{L}-1$. 


\section{Particle Swarm Optimization}

Particle Swarm Optimization (PSO) developed by James Kennedy and Russell Eberhart is bio-inspired algorithm aimed at the nature of behavior of bird flocking . In PSO algorithm each iteration, the "fitness" of the particle and the neighboring particles are observed and identifies the fit neighbor whose current position provides a improved solution to the problem. The algorithm first initializes a group of random particles called solutions and then proceeds to search for optima by modifying the generations. In each iteration, all the particles are updated by following two main "best" values. One is the best value or fitness value $p_{\text {best }}$ , that the particle has so far achieved. And other the global best value $\boldsymbol{g}_{\boldsymbol{b e s t}}$ that is identified by the particle swarm optimizer. After obtaining the two best values, the particle updates its velocity and position.

Each particle $n$ at time $t$ moves in n-dimensional space with respect to position $\left(x_{t}^{n}\right)$ and according to the velocity $\left(v_{t}^{n}\right)$ values. These values are very much dependent on the local best $\left(\overline{x_{t}^{n}}\right)$ and on the global best $\left(\overline{g_{t}^{n}}\right)$ values.

$v_{t+1}^{n}=\omega v_{t}^{n}+c_{1}\left(\overline{g_{t}^{n}}-x_{t}^{n}\right)+c_{2}\left(\overline{x_{t}^{n}}-x_{t}^{n}\right), \quad x_{t+1}^{n}=x_{t}^{n}+v_{t+1}^{n}$

The two learning factors $c_{1}$ and $c_{2}$ controls the effects of $p_{\text {best }}$ and $g_{\text {best }}$.

Random Inertia Weight $\mathrm{w}$ is the best strategy for better accuracy and efficiency of the algorithm.

$$
\begin{aligned}
& w=(0.5 * \text { rand }(1,1)+0.5) \\
& \mathrm{d}_{1}=\left|\overline{g_{t}^{n}}-x_{t}^{n}\right| ; \mathrm{d}_{2}=\left|\overline{x_{t}^{n}}-x_{t}^{n}\right| \text { i.e., } \quad \mathrm{d}_{1}=\left|g_{\text {best }}-x_{t}^{n}\right| ; \mathrm{d}_{2}=\left|p_{\text {best }}-x_{t}^{n}\right| \\
& d_{1} \text { - distance between the particles present position and its global best, and } \mathrm{d}_{2} \text { - distance }
\end{aligned}
$$
between the particles present position and the local best.

Current Best Performance Evaluation (CBPE) is the fitness value of a particle at that moment. To fit in to optimization problems the CBPE is normalized [8]. The normalized current best performance evaluation (NCBPE ) lying in $[0,1]$ is given by

$$
\mathrm{NCBPE}=\frac{C B P E-C B P E_{\min }}{C B P E_{\max }-C B P E_{\min }} \quad \text { where } C B P E_{\min } \text { is the estimated (or real) }
$$

minimum i.e., worst fitness attained so far. $C B P E_{\max }$ is the best fitness attained so far.

The probability distribution $p_{i}^{C}=\frac{h_{i}^{C}}{N}, \sum_{c=\{R, G, B\}}^{N} p_{i}^{c}=1$

$i$ - intensity level, $0 \leq i \leq L-1, \mathrm{C}$ - the RGB component of the image $\mathrm{C}=\{\mathrm{R}, \mathrm{G}, \mathrm{B}\}, \mathrm{N}$ the number of pixels in the image and $h_{i}^{c}$ - the count of pixels for intensity level $i$ in the component C.,$h_{i}^{c}$ - image histogram for each component $\mathrm{C}$.

The total mean or combined mean of each component of the image is $\sigma_{T}^{c}=\sum_{\substack{i=1 \\ c=\{R, G, B\}}}^{L} i p_{i}^{c}$

The algorithm that maximizes the between-class variance is considered to be the best method for deriving the optimal threshold which can be generally defined by:

$$
\sigma_{B}^{C^{2}}=\sum_{\substack{j=1 \\ C=\{R, G, B\}}}^{n} W_{j}^{C}\left(\mu_{j}^{C}-\mu_{T}^{C} \quad \text { where } \mathrm{j}\right. \text { - a specific class. }
$$


The probability of occurrence $W_{j}^{C}$ of classes $D_{1}^{C}, \ldots, D_{n}^{C}$ is , and the mean of each class $\mu_{j}^{c}$

is

$$
W_{j}^{C}=\left\{\begin{array}{c}
\sum_{\substack{i=1 \\
C=\{R, G, B\}}}^{t_{j}^{c}} p_{i}^{c}, \quad j=1 \\
\sum_{i=t_{j-1}^{c}+1}^{t_{j}^{c}} p_{i}^{c}, \quad 1<j<n \\
\begin{array}{c}
C=\{R, G, B\} \\
\sum_{i=t_{j-1}^{c}+1}^{L} p_{i}^{c}, \\
C=\{R, G, B\}
\end{array}
\end{array}\right.
$$

$$
, \mu_{j}^{C}=\left\{\begin{array}{cr}
\sum_{\substack{i=1 \\
t_{j}^{c}}} \frac{i p_{i}^{c}}{W_{j}^{c}}, \quad j=1 \\
\sum_{i=t_{j-1}^{c}+1}^{t_{j}^{c}} \frac{i p_{i}^{c}}{W_{j}^{c}}, \quad 1<j<n \\
C=\{R, G, B\} \\
\sum_{i=t_{j-1}^{c}+1}^{L} \frac{i p_{i}^{c}}{W_{j}^{c}}, \quad j=n \\
C=\{R, G, B\}
\end{array}\right.
$$

Now the complicated problem of multi-level thresholding is reformed into an optimization problem $[910,11,12]$ that searches the thresholds $t_{j}^{c}$ which maximizes RGB components objective functions or the fitness function of each,: $\psi^{c}={ }_{1<t_{j}^{c}<\cdots . .<t_{n-1}^{c}}^{\max _{B}} \mu_{B}^{C^{2}}\left(t_{j}^{c}\right)$

However, PSO also has some limitations of becoming trapped in local optimum. To overcome these limitations we use Fuzzy rules to the algorithm.

\section{Fuzzy Particle Swarm Optimization algorithm}

Fuzzy sets are a mathematical concept proposed by Prof. L. A. Zadeh in 1965 to express imprecision. A fuzzy rule $[16,17]$ is a basic unit for capturing knowledge in many fuzzy systems

This paper mainly focuses on the implementation of fuzzy If-Then rules by introducing a new velocity update approach for the particles in PSO and analyzes its effect on the particle's behavior. In the fuzzy system one of the output variable chosen is the scaling factor $c_{1}$ to control the effects of the particle's best value $\left(p_{\text {best }}\right)$. And another output variable is $c_{2}$, that controls the effects of the $g_{\text {best }}$, best value. To describe both the input and output parameters. $\mathrm{d}_{1}$ and $\mathrm{d}_{2}$, linguistic values "low", "medium", and "high" are used. Rules are framed in such a way that particles should reach the optimum and fitness should be acceptable. In other words if the parameters $d_{1}, d_{2}$ and NCBPE are low, then the particle is close to the optimum and hence the fitness value is suitable. For the search to be around global optimum $c_{1}$ and $c_{2}$ are given a high value.

Before an output is defuzzified all the fuzzy outputs of the system are aggregated with a union (max) operator. In this work, disjunctive system of rules is used for aggregation of all rules and for deffuzification centroid defuzzication method is applied to obtain the crisp output. In parameterized algorithms like PSO and Fuzzy PSO methods parameter values are to be chosen with care so that the method converges faster. The initial parameters taken for PSO and Fuzzy PSO algorithms are given in Table 1. 
TABLE 1 : INITIAL PARAMETERS OF THE PSO AND FUZZY PSO

\begin{tabular}{|l|l|l|}
\hline Parameters & PSO & Fuzzy PSO \\
\hline No. of iterations & 8 & 8 \\
\hline Population & 200 & 30 \\
\hline $\mathrm{c}_{1}$ & 1.5 & Fuzzy \\
\hline $\mathrm{c}_{2}$ & 1.5 & Fuzzy \\
\hline $\mathrm{W}$ & 1.2 & Chaotic random inertia weight \\
\hline $\mathrm{v}_{\max }$ & 2 & 2 \\
\hline $\mathrm{v}_{\min }$ & -2 & -2 \\
\hline $\mathrm{x}_{\max }$ & 255 & 255 \\
\hline $\mathrm{x}_{\min }$ & 0 & 0 \\
\hline
\end{tabular}

The proposed algorithm is implemented and tested on benchmark images. The performance and effectiveness of the proposed method is studied by comparing the results with PSO method. In all cases, the image segmentation approaches were programmed in MATLAB.

The stability of the algorithm is obtained using the index $\mathrm{SD}=\sqrt{\sum_{i=1}^{n} \frac{\left(\sigma_{i}-\mu\right)^{2}}{N}}$

where SD - standard deviation, $\sigma_{i}$ - best fitness value of the $\mathrm{i}^{\text {th }}$ iteration, $\mu$ is the average value of $\sigma_{\mathrm{i}}$ and $\mathrm{N}$ is the number of repetition of the algorithm Here $\mathrm{N}$ is taken as 20 .

To visually compare the segmented results of different test cases by Fuzzy PSO, the segmented images of Map, Airplane, Cameraman and Brain with 2 thresholds are given in Figure.1. As observed from the figure 2, images having 2 threshold segmentation using Fuzzy PSO have better detail than the images using PSO. The averages and the standard deviations for 20 trials are shown in Table 2 and Table 3 illustrates the optimum threshold values for PSO and FPSO methods used for segmentation of the images

\section{CONCLUSION}

Besides being a segmentation tool on its own, thresholding is frequently used as one of the steps in many advanced segmentation methods. This thesis presents a different way to approach image segmentation using fuzzy logic. .From experimental result values obtained it can be shown that the performance of the proposed method is better. Though it is observed that both PSO and Fuzzy PSO obtain similar results, it is to be noted that the fuzzy PSO algorithm obtains relatively better fitness solution. From Table 2, it is also observed that the stability of Fuzzy PSO is is high in comparison with PSO. Its noted from Figure 2 that Fuzzy PSO generates low standard deviation when compared with PSO. As a result Fuzzy PSO proves better than PSO in the test cases taken. To conclude it is seen that both PSO and Fuzzy PSO are valid and efficient methods with respect to their convergence 

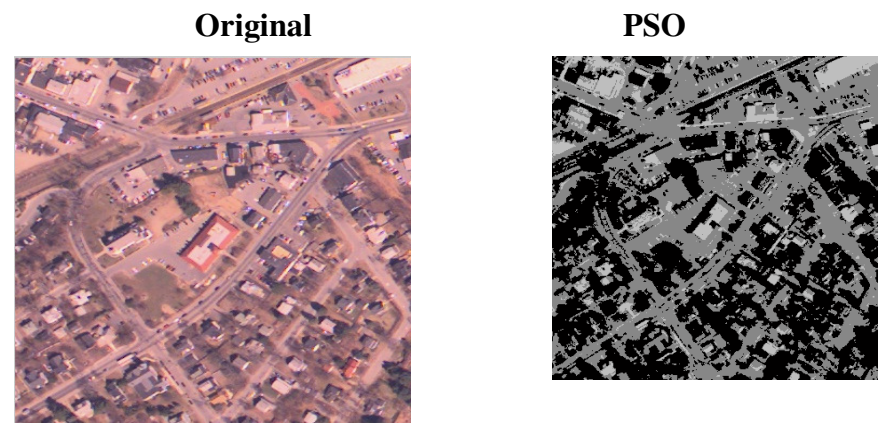

Fuzzy PSO
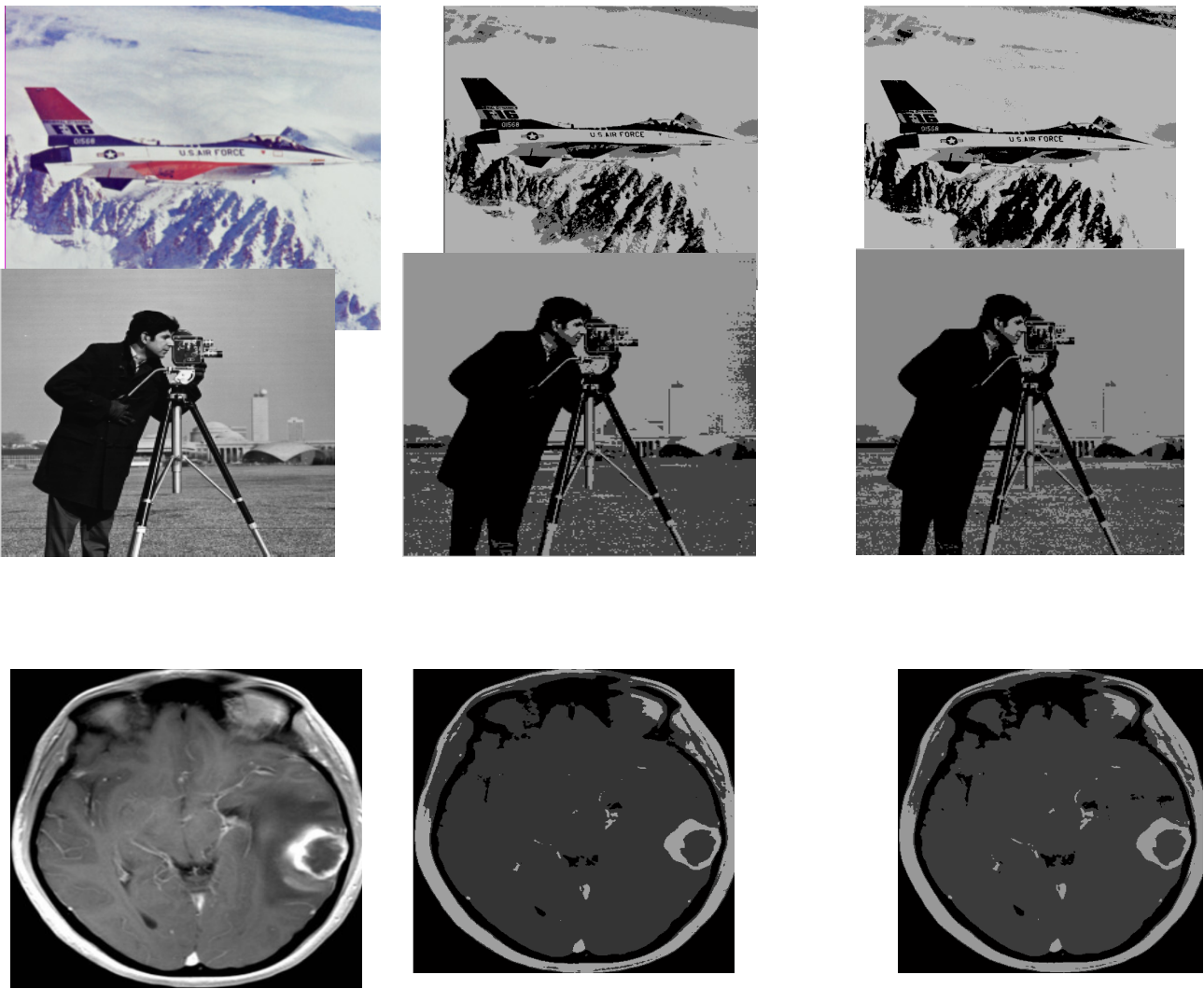

Fig. 1 Results of Segmentation images with 2 thresholds (top to bottom) 
TABLE 2 Average \pm SD values

\begin{tabular}{|c|c|c|c|}
\hline Test images & Thresholds & PSO & \begin{tabular}{|l|} 
Fuzzy PSO \\
\end{tabular} \\
\hline \multirow[t]{4}{*}{ Map } & 2 & $1036.4 \pm 25.1791$ & $1044.8 \pm 15.9858$ \\
\hline & 3 & $1077.8 \pm 23.7040$ & $1086.9 \pm 19.3836$ \\
\hline & 4 & $1110.1 \pm 19.0526$ & $1125.3 \pm 18.7904$ \\
\hline & 5 & $1161.1 \pm 19.7204$ & $1128.9 \pm 15.1968$ \\
\hline \multirow[t]{4}{*}{ Airplane } & 2 & $1937.4 \pm 18.2203$ & $1941.8 \pm 15.3735$ \\
\hline & 3 & $2001.2 \pm 23.3231$ & $2009.7 \pm 9.2991$ \\
\hline & 4 & $2044.5 \pm 12.8393$ & $2050.8 \pm 11.0279$ \\
\hline & 5 & $2069.1 \pm 14.2038$ & $2070.2 \pm 11.1513$ \\
\hline \multirow[t]{4}{*}{ Cameraman } & 2 & $3625.8 \pm 28.8799$ & $3634.1 \pm 15.3532$ \\
\hline & 3 & $3706.6 \pm 14.0528$ & $3708.7 \pm 9.2283$ \\
\hline & 4 & $3755.6 \pm 10.6688$ & $3758.3 \pm 10.2621$ \\
\hline & 5 & $3791.2 \pm 10.3591$ & $3787.4 \pm 9.4614$ \\
\hline \multirow[t]{4}{*}{ Brain } & 2 & $3551.7 \pm 18.9765$ & $3552.4 \pm 15.5485$ \\
\hline & 3 & $3720.8 \pm 14.3125$ & $3707.2 \pm 17.4187$ \\
\hline & 4 & $3817.3 \pm 18.2534$ & $3823.1 \pm 17.0028$ \\
\hline & 5 & $3872.0 \pm 10.8784$ & $3869.9 \pm 8.1954$ \\
\hline
\end{tabular}

Table 3 : Average threshold values

\begin{tabular}{|c|c|c|c|}
\hline Test Images & Thresholds & PSO & Fuzzy PSO \\
\hline \multirow[t]{4}{*}{ Мap } & 2 & 117164 & 116157 \\
\hline & 3 & $\begin{array}{lll}79 & 131 & 175\end{array}$ & $87 \quad 108 \quad 154$ \\
\hline & 4 & $\begin{array}{llll}60 & 112 & 142 & 162\end{array}$ & $\begin{array}{llll}13 & 139 & 176 & 184\end{array}$ \\
\hline & 5 & $\begin{array}{lllll}59 & 108 & 136 & 163 & 198\end{array}$ & $\begin{array}{lllll}89 & 124 & 144 & 198 & 232\end{array}$ \\
\hline \multirow[t]{2}{*}{ Airplane } & 2 & 118175 & 123181 \\
\hline & 3 & $\begin{array}{lll}74 & 123 & 185\end{array}$ & $65 \quad 116 \quad 156$ \\
\hline
\end{tabular}




\begin{tabular}{|c|c|c|c|}
\hline & 4 & $\begin{array}{llll}88 & 125 & 171 & 198\end{array}$ & $\begin{array}{llll}75 & 113 & 163 & 210\end{array}$ \\
\hline & 5 & $\begin{array}{lllll}86 & 116 & 142 & 178 & 202\end{array}$ & $\begin{array}{lllll}81 & 128 & 173 & 197 & 211\end{array}$ \\
\hline \multirow[t]{4}{*}{ Cameraman } & 2 & $78 \quad 145$ & $34 \quad 132$ \\
\hline & 3 & $\begin{array}{lll}52 & 108 & 142\end{array}$ & $\begin{array}{lll}57 & 123 & 150\end{array}$ \\
\hline & 4 & $\begin{array}{llll}53 & 111 & 152 & 198\end{array}$ & $\begin{array}{llll}51 & 121 & 157 & 172\end{array}$ \\
\hline & 5 & $\begin{array}{lllll}18 & 37 & 69 & 133 & 164\end{array}$ & $\begin{array}{lllll}29 & 87 & 131 & 162 & 199\end{array}$ \\
\hline \multirow[t]{4}{*}{ Brain } & 2 & $54 \quad 160$ & $60 \quad 152$ \\
\hline & 3 & $\begin{array}{lll}31 & 100 & 182\end{array}$ & $\begin{array}{lll}57 & 111 & 167\end{array}$ \\
\hline & 4 & $\begin{array}{llll}32 & 92 & 160 & 186\end{array}$ & $\begin{array}{llll}36 & 90 & 136 & 200\end{array}$ \\
\hline & 5 & $\begin{array}{lllll}35 & 76 & 103 & 141 & 172\end{array}$ & $\begin{array}{lllll}43 & 71 & 115 & 138 & 208\end{array}$ \\
\hline
\end{tabular}

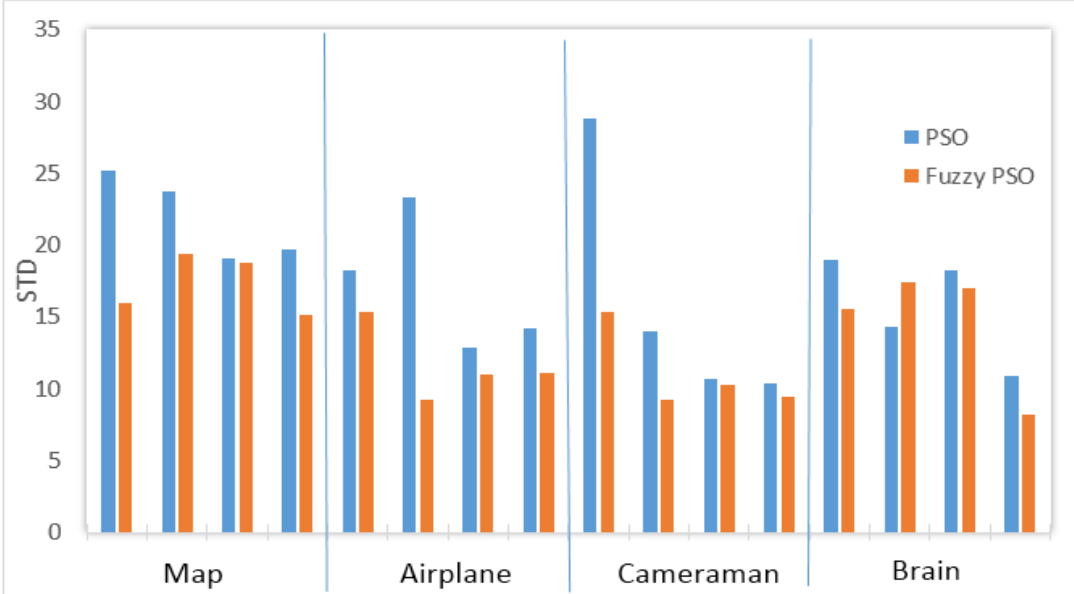

Fig 2 : SD values for both the algorithms for thresholds $2,3,4,5$ (from left to right)

\section{References}

[1] Brink, A. D.: Minimum spatial entropy threshold selection. IEEE Proceedings on VisionImage and Signal Processing, 142, 128-132 (1995)

[2] Kulkarni, R. V., \&Venayagamoorthy, G. K.: Bio-inspired algorithms for autonomousdeployment and localization of sensor nodes. IEEE Transactions, SMC-C40(6), 663-675 (2010)

[3] Sezgin, M., \&Sankur, B.: Survey over image thresholding techniques andquantitative Performanceevaluation. Journal of Electronic imaging, 3(1),146-168 (2004)

[4] M. Sonka, V. Hlavac and Boyle.R.: Image processing, analysis, and machine vision, Third edition, Thomson, USA(2008)

[5] Couceiro, M. S., Luz, J. M. A., Figueiredo, C. M., \& Ferreira, N. M. F.: Modeling and control of biologically inspired flying robots. Robotica (Vol. 30, pp. 107-121).CambridgeUniversity Press. 1 (2012)

[6] Pires, E. J. S., Machado, J. A. T., Oliveira, P. B. M., Cunha, J. B., \& Mendes, L.: Particle swarm optimization with fractional-order velocity. Journal on Nonlinear Dynamics, 61,295-301 (2010)

[7] J. C. Bansal, P. K. SinghMukeshSaraswat, AbhishekVerma, Shimpi Singh Jadon,Ajith Abraham.: Inertia Weight Strategies in Particle Swarm Optimization.IEEE,978-1-4577-1123-7/11 (2011) 
[8] AfshinGhanizadeh, SamanSinaie, Amir AtapourAbarghouei, and SitiMariyamShamsuddin.:A Fuzzy-Particle Swarm Optimization Based Algorithm for Solving Shortest Path Problem.IEEE,V6404 978-1-4244-6349-7/10 (2010)

[9] Otsu, N.: A threshold selection method from gray-level histograms. IEEE Transactions OnSystems, Man, Cybernetics, SMC-9, 62-66 (1979)

[10] Fogel, D. B.: Evolutionary computation: Toward a new philosophy of machineintelligence (Second ed.)Piscataway, NJ: IEEE Press (2000)

[11] Lai, C. C., \& Tseng, D. C.: A hybrid approach using Gaussian smoothing and genetic Algorithm for multilevel thresholding. International Journal of Hybrid Intelligent Systems,1(3), 143-152 (2004)

[12] Yin, P. Y. A fast scheme for optimal thresholding using genetic algorithms. Signal, Processing, 72, 8595 (1999)

[13] John Yen.: Fuzzy Logic and Control. Pearson Education(2003)

[14] H. J. Zimmerman.: Fuzzy set Theory and applications. Springer International(2006)

[15] Toshirpterano, KiyojiAsai, MichioSugeno.: Fuzzy Systems Theory and its applications, Academic press(2005)

[16] J. S. R. Jang, C. T. Sun, E. Mizutani.: Neuro - Fuzzy and soft computing. Prentice - Hall(1997)

[17] WitoldPedryez, Fernando Gomide.: An Introduction to Fuzzy Sets-Analysis and Design.Prentice Hall of India(2005)

[18] Kapur, J. N., Sahoo, P. K., \& Wong, A. K. C.: A new method for gray-level picture thresholding using the entropy of thehistogram. Computer Vision Graphics ImageProcessing. 2, 273-285 (1985)

[19] Cheng, H. D., Chen, J., \& Li, J.: Threshold selection based on fuzzy c-partition entropy approach. Pattern Recognition, 31, 857-870 (1998)

[20] Saha, P. K., \&Udupa, J. K.: Optimum image thresholdingvia class uncertainty and region homogeneity.IEEE Transactions on Pattern Analysis and achine Intelligence, 23, 689-706 (2001)

[21] Tobias, O. j., \&Seara, R.: Image segmentation by histogram thresholding using fuzzy sets. IEEE Transactions on Image Processing, 11, 1457-1465 (2002)

[22] Hu, Q., Hou, Z., \&Nowinski, W.: Supervised range-constrained thresholding.IEEE Transactions on Image Processing, 15, 228-240 (2006)

[23] Floreano, D., \&Mattiussi, C.: Bio-inspired artificial intelligence: Theories, methods, and technologies. Cambridge, MA: MIT Press(2008)

[24] M. H. NorooziBeyrami, and M. R. Meybodi.:Improving Particle Swarm Optimization using Fuzzy Logic. Proceedings of the Second Iranian Data Mining Conference. AmirkabirUniversity of Technology, Tehran, Iran, pp. 108-120 (2008)

[25] K. P. Wang, L. Huang, C. G. Zhou and W. Pang.: Particle swarm optimization for traveling salesman problem. Proceedings of International Conference on Machine Learning andCybernetics, pp. 1583-1585 (2003)

[26] Hammouche, K., Diaf, M., \&Siarry, P.: A comparative study of various meta- heuristic techniques applied to the multilevel thresholding problem. Engineering Applications of Artificial Intelligence, 23, 676-688(2010)

[27] Jiang, M., Luo, Y. P., \& Yang, S. Y.: Stochastic convergence analysis and parameter selection of the standard particle swarm optimization algorithm. Information Processing Letters, 102(1), 816(2007)

[28] PedramGhamisi, Micael S. Couceiro, Jon AtliBenediktsson, Nuno M. F. Ferreira.: An Efficient method for segmentation of images based on fractional calculus and natural selection. Expert Systems with Applications 39, 12407-12417(2012)

[29] Couceiro, M. S., Ferreira, N. M. F., \& Machado, J. A. T.: In Fractional orderDarwinian Particle Swarm Optimization, FSS'11 - Symposium on fractional signals and systems, November 4-5, Coimbra, Portugal(2011)

[30] Couceiro, M. S., Ferreira, N. M. F., \& Machado, J. A. T.: Application of fractional algorithms in the control of a robotic bird. Journal of Communications in Nonlinear Science and Numerical Simulation - Special Issue, 15(4), 895-910(2010) 
[31] Tillett, j., Rao, T. M., Sahin, F., Rao, R., \& Brockport, S.: Darwinian ParticleSwarm Optimization. In Proceedings of the 2nd Indian international conference on artificial intelligence (pp. 14741487)(2005)

[32] Naidu, M.; Kumar, P.R.; Chiranjeevi, K.: Shannon and fuzzy entropy based evolutionary image thresholding for image segmentation. Alex. Eng. J 57(3),1643-1655 (2018)

[33] Suresh, S.; Lal, S.: An efficient cuckoo search algorithm based multilevel thresholding forsegmentation of satellite images using different objective functions. Expert Syst. Appl. 58, 184209(2016)

[34] .C, Rama Sushil \&M. L. Garg.: An Improved PSO-Based Multilevel Image Segmentation Technique Using Minimum Cross-Entropy Thresholding.Arabian Journal for Science and Engineering volume 44, pages3005-3020 (2019)

[35] K.P.Baby Resma, Madhu S.Nair.: Multilevel thresholding for image segmentation using Krill Herd Optimization Algorithm. Journal of King Saud University - Computer and Information Sciences , Pages 528-541 (2021)

[36] Kennedy, J.; Eberhart, R. "Particle Swarm Optimization". Proceedings of IEEE International Conference on Neural Networks. IV. pp. 1942-1948, (1995). 